\title{
Dinâmica estacional de uma população de linophneles dlarlingi, numa área endêmica de malária no Amazonas
}

Jack Hayes (")

J. Derek Charlwood ( ${ }^{\star}$ )

\begin{abstract}
Resumo
A atividade cie picadas do Anopheles darlingi foi estudada por mais de um ano numa área endêmica de maläria na BR-174 (Manaus/Boa Vista). Esta atividade foi documentada para demonstrar as mudanças estacionuis na população e variação do ciclo nas estações do ano. São apresentadas as coletas mostrando o ciclo de picada dentro e fora das casas. O modelo foi constante na área estudada e verificou-se que a população de $\mathbf{A}$. darlingi aumenta na estação seca. Com o aumento das chuvas há um declínio na população de anófeles e esta diminuição corresponde a um decréscimo estacional similar ao número de casos de infecção malárica.
\end{abstract}

\section{INTRODUÇÃ̃o}

A incidência da malária no Amazonas mostrava um aparente declínico até a época do inicio das aberturas das estradas. Um relato recente deste fato é dado no trabalho Ferraroni \& Hayes (1978). O impacto que a malária causa nos colonizadores instalados na rodovia recentemente aberta (BR-174, Manaus/Boa Vista) é apresentado no trabalho Hayes \& Ferraroni (1978). Este trabalho representa os dados de mais de um ano de observação sobre o comportamento de picada do Anopheles darlingi num local situado no $\mathrm{Km} 137$ da rodovia BR 174 .

\section{Materiais E MÉTodos}

Em janeiro de 1977, iniciou-se a coleta usando as técnicas padronizadas pela OMS (1975) . Como iscas, foram usadas as porções médias e terminais dos membros inferiores de incividuos e as fêmeas eram capturadas quando ali pousavam. As iscas foram coiocadas periciomiciliares.

Os métodos de capturas recomendado pela OMS (1975) provam ser inadequacios e foram substituídos pela captuira das fêmeas, quando estas pousavam nas iscas deitadas sobre redes. Uma lanterna fơi colocada atrás da rede (entre a rede e a parede) e o coletor fazia as capturas das fêmeas darlingi enquanto elas se alimentavam no lado da rede onde havia sombra.

Os mosquitos capturados eram marcados em intervalos de 15 minutos e agrupados de hora em hora. Todos os capturadores que não estavam em serviço usavam mosquiteiros. Sempre havia 4 ou 5 coletores e 1 isca .

Nos primeiros meses de 1977, as coletas foram realizadas em duas localidades. Numa casa detetizada coietou-se amostras peridomiciliares e outras foram coletadas dentro de uma casa não detetizada, que fica a 500 metros no sentido norte da primeira, ou seja, do km 137. Em maio, a SUCAM borrifou com DDT o interior da casa e, após esta data, foram iniciadas capturas no seu interior e também no laboratório de campo, recentemente construido (que não foi dedetizado). Realizou-se capturas fora das casas em 4 ocasiões no km 137 . Somente 2 capturas dentro e fora de casa, ao mesmo tempo, foram realizadas no km 190.

Os dados pluviométricos dos locais foram os seguintes: Reserva Ducke (km 26 da AM10. Manaus/Itacoatiara) e no acampamento militar no km 200 da BR 174 (Manaus/Boa Vis. ta) .

\section{Resultados E discussão}

A incidência de malária no Amazonas aumenta no final da estação seca (Ferraroni \& Hayes, 1978) e a população de A. darlingi acompanha a mesma curva, porém, um pouco mais a frente, como ocorreu no $\mathrm{km} \mathrm{137.} \mathrm{A}$ população da $A$. darlingi apresentou seu menor pico em junho (gráfico I) e cresceu gradativa.

(*) - Instituto Nacional de Pesquisas da Amazônia, Manaus. 
menie, atingindo um pico no final de setembro. As coletas foram realizadas mensalmente, exceto no momento de uma rápida troca na população de mosquitos, uma vez que as capturas eram realizadas cada 2 ou 3 semanas. Existe um relacionamento grande entre a chuva e A. darlingi. Assim sendo, é evidente, embora não tendo sido possível coletar os dados pluviométricos no $\mathrm{km} \mathrm{137}$, tendo em vista que esses dados foram obtidos no $\mathrm{km} \mathrm{200,} \mathrm{ou} \mathrm{seja,}$ 63 km de distância no sentido norte e na Reserva Ducke, a uma distância pouco mais de $100 \mathrm{~km}$ no sentido sul do local da coleta. Existem grandes variações de chuvas dentro de um mesmo mês numa comparação anual. O totai de chuva em 1977 na Reserva Ducke foi de $2434,4 \mathrm{~mm}$ e no $\mathrm{km} \mathrm{200,} 2200 \mathrm{~mm}$ (tabela I). As estações situam-se a mais de $200 \mathrm{~km}$ de distância e, ao compararmos o total de chuvas em ambas, verificamos uma similaridade bem marcante e, principalmente, nos últimos 3 anos, verificamos que a estação da Reserva Ducke tem somente $11 \mathrm{~mm}$ a mais de signifi cância, enquanto que a estação do km 200 tem $1,078 \mathrm{~mm}$ abaixo da média. A variação pluvio. métrica mensal é claramente evidente na estação seca; no km 200, por exemplo, a menor quantidade de chuva foi de $15,5 \mathrm{~mm}$ em novem-

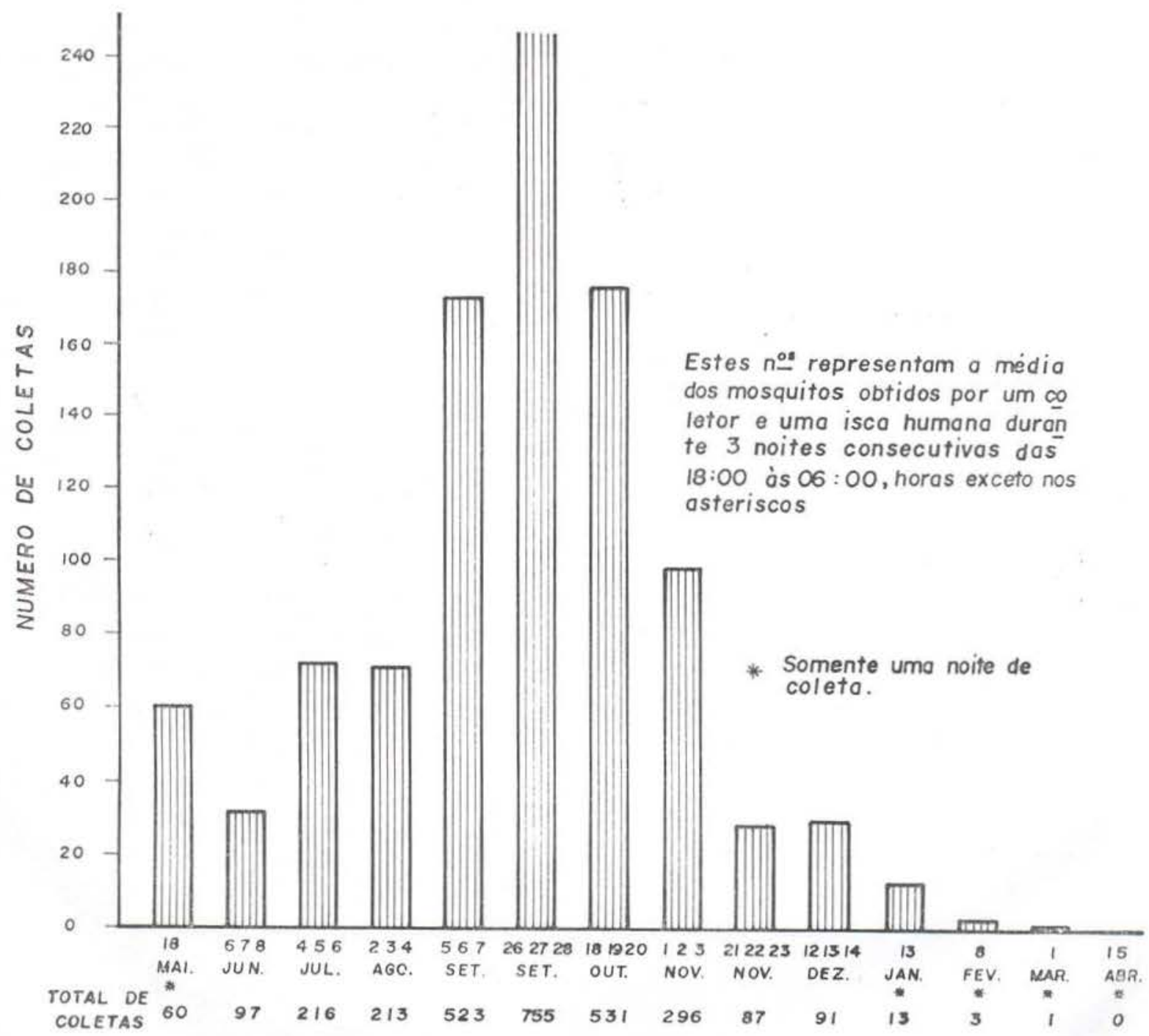

GRAFICO I - Mudança sazonal na população de Anopheles darlingi picando o homem (isca humana), no período de maio/77 a março/78 no $\mathrm{Km} 137$ da BR-174, Manaus - Boa Vista. 
bro de 1977, enquanto que para o mesmo mês, no ano de 1976, foi de $170,0 \mathrm{~mm}$. Os dados sobre malária nesta área nos últimos 4 anos tornam bem claro que o pico sempre ocorre no final da estação seca (Ferraroni \& Hayes, 1978) .

Em nossa opinião, a geografia da área influencia diretamente o relacionamento das chuvas com as populações de mosquitos. Este local é alto, e as chuvas são suficientes para carregar as larvas. As chuvas, quando intensas, fazem crescer o nivel das águas nos igarapés para 2 a 3 metros num espaço de tempo de 2 a 6 horas, como observamos várias vezes. Isto tende a carregar as larvas e os ovos. Nas áreas baixas (várzea) ocorre o oposto, as águas das chuvas enchem as lagoas e depressōes secas, as quais formam mais criadouros (Guedes et al. 1953 e Silva, 1975). Nas áreas da BR 174, as mesmas condições ideais para o desenvolvimento das larvas ocorrem nas estaçōes secas, quando os igarapés estã? estacionados e não ocorrem as grandes enxurradas. Desta maneira, a população de $A$. darlingi no km 137 atinge um pico no final de setembro e vai declinando gradualmente por todo o mês de novembro. O número coletado em dezembro (as 3 últimas noites consecutivas de captura) apresentou uma média de 30 pe noite. Os números coletados em janeiro e meses seguintes foram tão baixos que houve captura somente em uma noite. Somente 3 mosquitos foram coletados em fevereiro e $1 \mathrm{em}$. março, quando foi concluído este trabalho (gráfico 1). A variação no ciclo de picada do darlingi nesta área é mínima (gráficos 11 c III), existindo uma pequena diferença no modo padrăo da picada.

As picadas apresentam um pequeno pico ao escurecer durante a primeira hora, seguido por uma queda na segunda hora. A partir deste momento, as picadas aumentam continuamente, atingido um máximo entre $21: 30$ e 22:30 horas; deste ponto inicia-se um declínio gradual até o nascer do sol. Suspeitamos que durante a primeira hora de atividade, às fêmeas tenham se alimentado previamente. Os resultados preliminares das dissecções de ovários não estão ainda concluídos, mas a paridade pode ser encontrada em todo o momento durante a noite. A variação do $A$. darlingi picando o homem, ocorre geralmente de acordo com a posição geográfica (Charlwood \& Hayes, 1978). No entanto, é pouco significativa para esta área no $\mathrm{km} 137$. Não temos um pico distinto entre as picadas nas noites de 2, 3 e 4 de agosto (gráfico II). Cada coleta apresenta um padrão típico. O pico das picadas dentro de casa no km 199 é o mesmo do km 137. Todavia, o pico fora de casa para as mesmas noites ocorrem freqüentemente à meia noite (gráfico iV). É muito importante verificar que năo se pode fazer deduções precisas sobre as populações, a não ser quando se realiza uma coleta completa durante a noite ( 12 horas). O ideal seria fazer uma média das coletas durante várias noites consecutivas.

Em algumas áreas, um grande número de A. darlingi foi coletado após a meia noite (gráfico IV) (Ferraroni \& Hayes, 1977 e Charlwood \& Hayes, 1978).

Ao determinar o crescimento estacional da população de $A$. darlingi, usando somente capturas ao anoitecer, pode se obter um resultado não correto, na detecção das trocas estacionais (sazonal) na variação geográfica.

Dados mostrando a população de darlingi na primavera ou nos meados da estação chuvosa, podem ser encontrados em Charlwood \& Hayes (1978). Esses dados mostram um grande número de mosquitos nessa época do ano, mesmo sendo esta coleta realizada em uma casa não detetizada, numa distância de $1 / 2 \mathrm{~km}$ a norte do $\mathrm{km} 137$. Um número médio de 225 mosquitos por noite foram coletados em 29, 30 e 31 de março de 1976 . Este número alto citado aqui, e aquele coletado no $\mathrm{km} 199 \mathrm{em}$ novembro de 1977, foram devidos ao número de hospedeiros (homem) disponível nos dois locais, para abastecer as necessidades sugadoras dos mosquitos que foram contados nas diferenças populacionais de $A$. darlingi. Ainda em cada área as populações de $A$. darlingi seguiram o mesmo padrão estacional demonstrado no $\mathrm{km} 137$.

Início da coleta através da isca humana, apresentou um grande problema. As observações foram iniciadas em outubro de 1976, quando o primeiro autor contraiu a malária por 
Plasmodium falciparum. Novamente, outros dois capturadores contrairam malária, estes não estavam usando profiláticos e um dos casos foi fatal, sendo o óbito causado por malária falciparum. Após este fato, nenhum dos auxiliares queria participar das excursões, a não ser fazendo uso de quimioprofiláticos

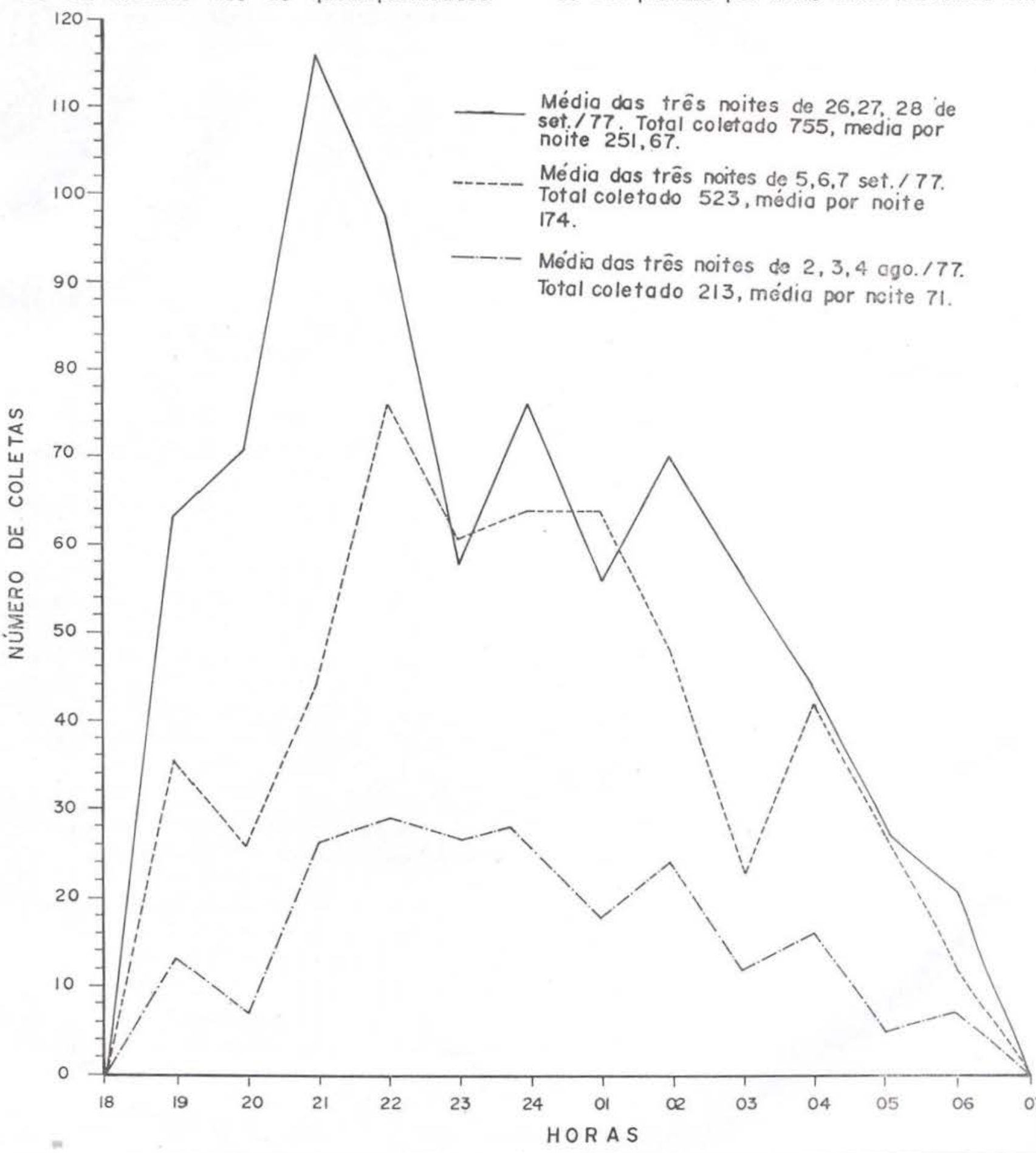

GRAFICO II - Anopheles darlingi, capturados em isça humana, dentro de casa no início da estaçăo seca, agosto e setembro de 1977, no Km 153 da BR-174, Manaus-Boe Vista.
(Cloroquina/primaquina). Assim sendo, nesta localidade, não houve mais casos de malária na equipe que participava do trabalho. No en tanto, em outubro do mesmo ano, todos os 4 coletores de mosquitos contraíram malária resistente às cloroquinas no $\mathrm{km} 190$, onde mais de 300 picadas por noite eram recebidas em
Média das três noites de 26,27,28 de noite 251,67 .

Média das três noites de $5,6,7$ set. $/ 77$.

Total coletado 523, média por noite

Média das três noites de $2,3,4 \mathrm{ago} .177$

total coletado 213 , média por ncite 71 
cada coletor. Desta maneira, sugere-se que o profilático age de maneira relativa, dependendo da quantidade de esporozóitos injetados no organismo pelos anofeles.

\section{CONCLUSÃo}

Como foi citado por Hayes \& Charlwood (1978), o A. darlingi constantemente pousa fora de casa, praticamente em todas as locali- dades onde foram realizadas as coletas. Eles evitam o DDT borrifado nas superfícies; isto é evidente pela observação direta e a continuação de um número altơ de casos de infecção malárica nos 4 últimos anos, apesar do DDT ser espaça de 6 em 6 meses. O ciclo de picadas do $A$. darlingi demostra a evidência do contato com o homem durante a noite. A coincidência do meio geográfico com a época

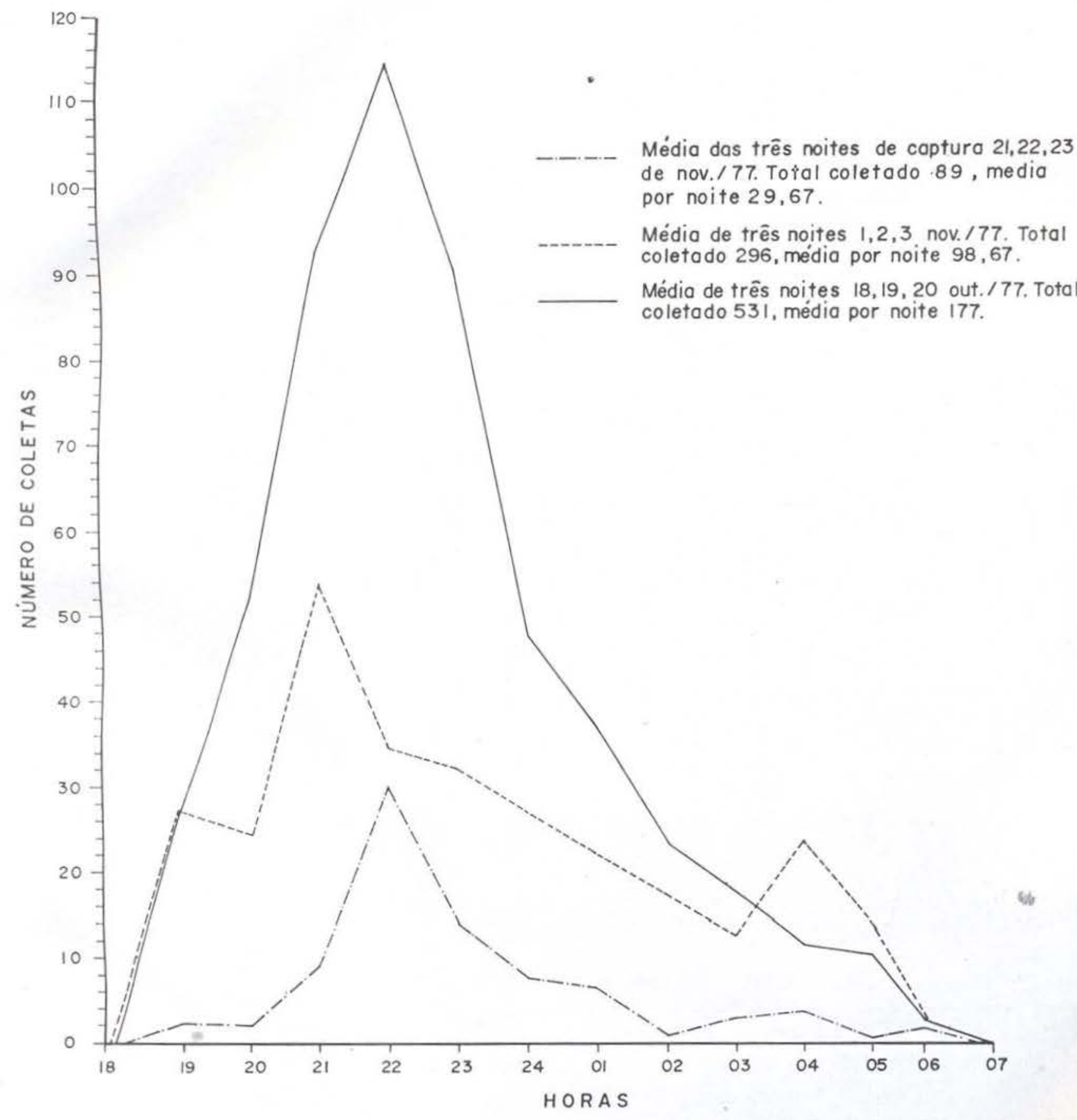

GRAFICO III - Anopheles darlingi, capturados em isca humana, dentro de casa no final da estação seca, outubro e novembio de 1977, no Km 153 da BR-174, Manaus-Boa Vista. 
das chuvas faz com que a população mais alta apareça no final da estação seca. Recomendações para o controle da malária e A. darlingi nesta área foram apresentados por Hayes \& Ferraroni (1978). Faz-se necessário um maior conhecimento sobre esse vetor, especialmente sobre a comportamento do adulto, assim como sua biologia larval.
Trabalhos anteriores citam o A. darling sugando outros animais e indicam serem menos antropofílicos (Deane, 1948). No km 137, não capturamos darlingi usando bois como isca. Entretanto, a $1 / 2 \mathrm{~km}$ no sentido norte do $\mathrm{km} \mathrm{137,} \mathrm{o} \mathrm{darlingi} \mathrm{foi} \mathrm{coletado} \mathrm{sugando}$ cães, assim como no Aripuanã em Mato Grosso. O trabalho de Deane (1948) e Galvão et al.

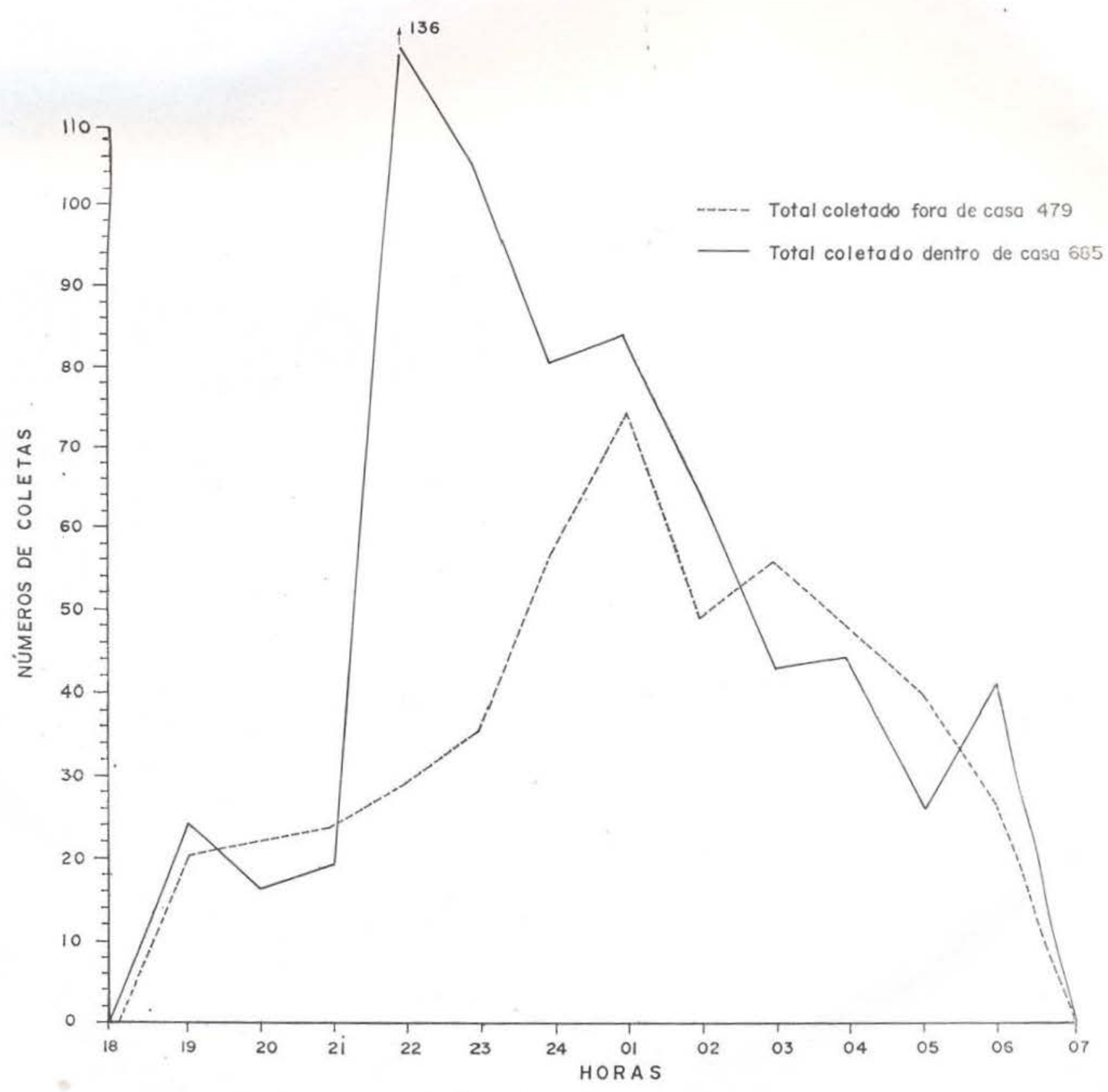

GRAFICO IV - Captura de Anopheles darlingi dentro dentro e fora de casa no Km 199 da BR-174 no período de $28 / 10 / 77$ - Número expresso por homem/horas. 
TABELA I - MM de chuva na Reserva Ducke, Km 26 da estrada AM-10 (Ma naus/Itacoatiara) e Km 200 da estrada BR-174 (Manaus/Boa Vista) no período de janeíro de 1974 a dezembro de 1977

ESTRADA E KM
MESES DO ANO

\begin{tabular}{|c|c|c|c|c|c|c|c|c|c|c|c|c|c|}
\hline AM- $10 \mathrm{Km} 26$ & Jan. & Fev. & Mar. & Abr. & Maio & Junho & Julho & Agosto & Set. & Out. & Nov. & Dez. & Total \\
\hline \multicolumn{14}{|l|}{ A NO } \\
\hline 1974 & 142.0 & 248.3 & 264.5 & 337.1 & 271.5 & 181.8 & 180.4 & 73.2 & 114.6 & 47.6 & 209.8 & 202.8 & 2273.60 \\
\hline 1975 & 221.4 & 186.1 & 269.9 & 321.0 & 305.3 & 129.6 & 121.0 & 88.2 & 40.5 & 290.7 & 169.7 & 207.6 & 2351.00 \\
\hline 1976 & 326.5 & 572.2 & 401.8 & 312.0 & 209.4 & 89.5 & 122.3 & 51.8 & 6.8 & 203.0 & 77.4 & 262.7 & 2635.48 \\
\hline TOTAL & 803.9 & 1231.8 & 1318.0 & 1361.3 & 1054.0 & 499.3 & 503.6 & 236.2 & 327.7 & 817.1 & 606.5 & 935.5 & \\
\hline MEDIA & 200.98 & 307.95 & 329.5 & 340.33 & 263.5 & 124.83 & 125.9 & 59.05 & 81.93 & 204.28 & 151.63 & 233.8 & \\
\hline
\end{tabular}

BR-174-KM 200

$\triangle \mathrm{NO}$

\begin{tabular}{|c|c|c|c|c|c|c|c|c|c|c|c|c|c|}
\hline 1974 & 283.1 & 634.4 & 432.8 & 535.1 & 346.7 & 440.0 & 217.5 & 214.5 & 240.7 & 161.0 & 95.3 & 471.8 & 4072.90 \\
\hline 1975 & 368.2 & 196.6 & 364.1 & 482.7 & 536.3 & 320.0 & 221.6 & 240.6 & 91.6 & 239.7 & 71.8 & 172.7 & 3305.90 \\
\hline 1976 & 358.5 & 182.4 & 492.0 & 466.1 & 482.0 & 553.0 & 325.4 & 236.0 & 108.3 & 48.3 & 170.0 & 170.0 & 3592.00 \\
\hline 1977 & 200.2 & 198.3 & 355.5 & 373.6 & 332.0 & 181.5 & 59.3 & 61.5 & 248.7 & 76.0 & 15.5 & 98.5 & 2200.60 \\
\hline TOTAL & 1209.0 & 1211,7 & 1644.4 & 1857.5 & 1697.0 & 1494.5 & 823.2 & 952.6 & 639.3 & 525.0 & 352.6 & 913.0 & \\
\hline MEDIA & 302.25 & 302.9 & 411.10 & 464.38 & 424.25 & 373.63 & 205.8 & 188.15 & 159.83 & 131.25 & 88.15 & 228.25 & \\
\hline
\end{tabular}


no Pará (1942) apresentam um quadro diferente de $A$. darlingi nos anos de 1940 , rotineiramente coletados pousando nas casas, e sugando durante o dia.

Duas vezes coletamos fêmeas picando após as 8:00 horas da manhã, mas estas ocasiōes, foram possíveis devido elas estarem em armadilhas no laboratório. De uma grande variedade de modelos no comportamento de pouso e de picadas, parece que esta espécie tem sofrido algumas mudanças nos últimos 30-40 anos, em resposta aos inseticidas em populações isoladas na vasta Amazônia. Nes. tas regiões, a biologia tem evoluído, e trocas pode ter ocorrido. É evidente que o darlingi é o único vetor que pica dentro das casas e somente 2 Anopheles oswaldoi foram coletados no decorreh deste estudo, e eles se encontravam nas coletas peridomiciliaris. Parece ser evidente que o controle do darlingi deve ser procedido pelo estudo do seu comportamento.

\section{SUMMARY}

The biting activity of Anopheles darlingi was studied for over a year in an endemic area of malaria - BR-174 (Manaus/Boa Vista). The biting activity was documented to show seasonal changes in population and variation in the cycle with changes of season. Data is presented for the outdoor biting cycle and indoor biting cycle collections. Patterns remained constant within the study area and the population of A. darlingi increases in dry season. Intense rainfall is accredited the decline in population - this decline corresponds to a similar seasonal decrease in the number of malaria cases.

\section{BIBLIOGRAFIA}

ChARLwood, J.D. \& HAYEs, J.

1978 - Variações geográficas no ciclo de picada do Anopheles darlingi Root no Brasil. Acta Amazonica, 8(4) : 601-603.
Deane, L.M. : CAusey, O.E. \& DeAne, M.P.

1948 - "Notas sobre a distribuição e a biologia dos anofelinos das regiôes nordestina e Amazônica do Brasil". Revista do Serviço Especial de Saúde Pública. 1(4) : 827-965.

FerraroNi, J.J. \& HAYES, J.

1977 - Estudo sobre um surto de malária entre os indios Mayongong e Sanomã (Norte de Roraima) Acta Amazonica, 7 (3) : 401-406.

1978 - Aspectos epidemiológicos da malária no Amazonas. Acta Amazonica. (No prele).

Guedes, A. dA S.; Freitas, J.R. de \& Xavier, S.H.

1953 - Contribuição ao conhecimento da distribuição geográfica dos anofelinos e algumas observações sobre a biologia do Anopheles darlingi Root, 1926, no Esiado de Minas Gerais, Brasil, Revista Brasileira de Malariologia e Doenças Tropicais, 5(2) : 157-165.

HAYES, J. \& FERRARONI, J.J.

1978 - Ocorrência de melária numa estrada recém construída no Amazonas. Acta Amazonica 8(3) : 397-407.

Galvão, A.L.A.; Damasceno, R.G. \& Marques, A.P. 1942 - Algumas observaçōes sobre a biologia dos anofelinos da importância epidemiológica de Belém, Pará. Arquivos de Hi. giene. V. $12: 51-111$.

Suys, R.A. DA

1975 - Malária clínico epidemiológico, na recente colonização Agrícola de Região de Buriticupu, no Estado do Maranhão. Tese de Mestrado - Doenças Infeccio. sas e Parasitárias da Faculdade de Medicina da Universidade Federal do Rio de Janeiro.

WORLD HEALTh ORganization

1975 - Manual on Practical Entomology in Malaria, Part II Methods and Techniques WHO - Geneva.

(Aceito para publicaçāo em 06/05/78) 